
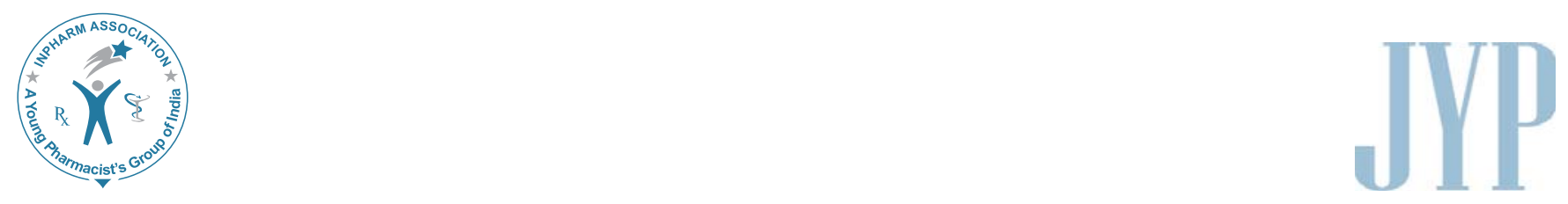

\title{
In-silico Comparative Study and Quantitative Structure-activity Relationship Analysis of Some Structural and Physiochemical Descriptors of Elvitegravir Analogs
}

\author{
Satpathy R, Ghosh $\mathbf{S}^{1}$ \\ Department of Biotechnology, MIRC LAB, MITS Engineering College, Rayagada, Odisha, \\ ${ }^{1}$ Amity University, Noida, Uttar Pradesh, India \\ Address for correspondence: Dr. Raghunath Satpathy; E-mail: rnsatpathy@gmail.com
}

\begin{abstract}
Elvitegravir is a new-generation drug which acts as an integrase inhibitor of the HIV virus. The potential inhibition has been tested from the clinical trial data. Here the work basically deals with the quantitative structure-activity relationship (QSAR) analysis by considering some of the physiochemical descriptors like molecular weight, logP, molar volume, and structural descriptors like Winers index, and molecular topological index of the drug analogs. The descriptors were calculated from the E-Dragon server and the multiple linear regression equation models were built by using Minitab tools. The different combinations of structural and physiochemical descriptors were considered for model derivation. The best three models were chosen by observing high R-Sq value, high F-value and low residual errors. The $P$ values (regression) for the three models indicates the significance of the consi dered descriptors. The overall results obtained with these model suggest that for this perticular drug the activity is dependent on physiochemical descriptors.
\end{abstract}

Key words: Integrase inhibitor, multiple regression analysis, physiochemical descriptor, quantitative structure-activity relationship, structural descriptor

\section{INTRODUCTION}

According to the IAVI (International AIDS Vaccine Initiative) report almost 7000 people are newly infected with HIV, and the death rate per day is around 6000. Still it

\begin{tabular}{|l|l|}
\hline \multicolumn{2}{|c|}{ Access this article online } \\
\hline Quick Response Code: & \\
\hline ⿴囗口 & Website: \\
\hline & www.jyoungpharm.in \\
& DOI: \\
\hline
\end{tabular}

is in a pandemic state hence currently a great international concern. ${ }^{[1]}$ Many potential drug molecules have been discovered to inhibit a specific part of the viral lifecycle as a current approach to control the disease. ${ }^{[2]}$ An area of much recent progress has been that of HIV integrase inhibitor design. Integrase is an essential enzyme for viral replication, and it has no human homolog. ${ }^{[3]}$ Integrase catalyzes the insertion of reverse-transcribed viral cDNA into the host cell genome via a multi-step process. The first step in integration occurs in the host cell cytosol and is referred to as 3' end processing. During this step, integrase cleaves a dinucleotide from each viral DNA terminus at a conserved sequence, yielding two reactive 3 ' 
hydroxyl groups. After this processing step a number of viral and cellular proteins form a complex which is known as pre-integration complex (PIC), and then migrates to the nucleus. Within the nucleus the reactive hydroxyl groups are utilized in nucleophilic attack upon the host cell genome, a process known as strand transfer. ${ }^{[4]}$ Elvitegravir is a potential drug for HIV integrase enzyme inhibitor which is in its Phase III clinical trials. ${ }^{[5,6]}$

To analyze different potential drug molecules the quantitative structure-activity relationship (QSAR) method is a useful approach. QSAR is basically used to study the biological activities with various properties associated with the structures, which is helpful to explain how structural features in a drug molecule influence the biological activities. Also a successful in silico-based QSAR analysis provides the advantages of higher speed and lower costs for bioactivity evaluation as compared to experimental testing. ${ }^{[7]}$ Therefore, correlating the physiochemical properties or structural features of the integrase inhibitor compounds with their biological activity will surely provide useful information for the design of new HIV drugs. To address this issue, an in silico approach has been adopted to calculate some selected physiochemical and structural descriptors of Elvitegravir analogs. Quantitative structural activity relationship study has been done by taking combinations of different physiological and structural descriptors by multiple linear regressions analysis to figure out the major molecular factor as associated with the activity of the drug molecule.

\section{MATERIALS AND METHODS}

All 26 analogs of the drug molecule Elvitegravir along with the IC50 value were obtained from the literature ${ }^{[8]}$ and corresponding $\log$ IC50 values were calculated. The derivatives of the Elvitegravir molecules were drawn in Marvin sketch 5.0 tools. ${ }^{\left[{ }^{[9]}\right.}$ Then the molecules were subjected to energy minimization by Prodrg server. ${ }^{[10]}$ Prodrg is an online server where the energy minimization of the molecule was performed by using Gromos 96 force field. The various descriptors considered for the present work were molecular weight (MW), molar volume (MV), $\log \mathrm{P}$ (Octanol/Water partition co efficient) as physiochemical descriptors, and molecular topological index (MTI), Wiener index (WI), and total E-state topological parameter (TIE) as structural descriptors for the molecules. All these descriptors were calculated by the E-Dragon server. ${ }^{[1]]}$ The different combinations of the above two types of descriptors were subjected to multiple regression analysis by MINITAB 14 software. ${ }^{[12]}$ For the best model selection various parameters like high $\mathrm{F}$ value, R-Sq and $P$ value were chosen from regression analysis and equations were derived. From the equations the predicted and experimental $\log$ IC50 values were compared.

\section{RESULTS}

In the present study an attempt has been made to develop the best QSAR model to explain the correlation between the combined effect of physiochemical and structural

Table 1: The Elvitegravir drug analogs along with their Log IC50 value

Structure Log IC50 value
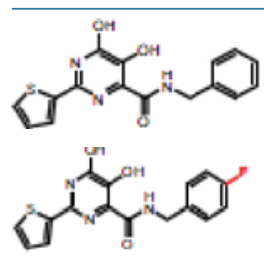

1.90
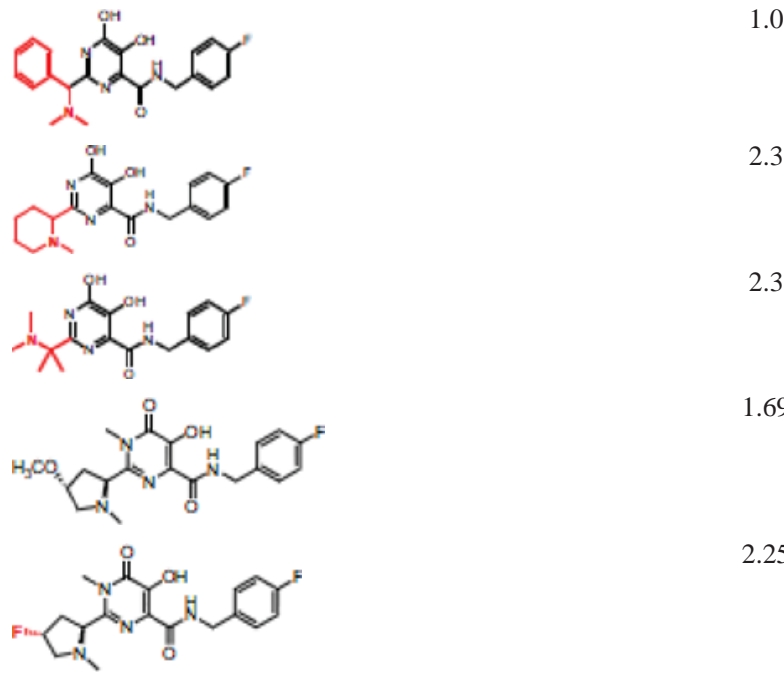

2.25

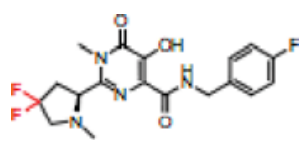

1.30

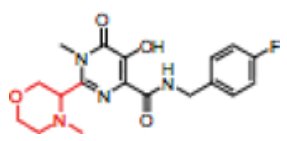

1.47
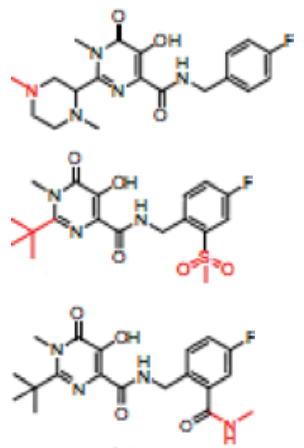

1.30

Table 1 contd. 
Table 1 Contd...

Structure
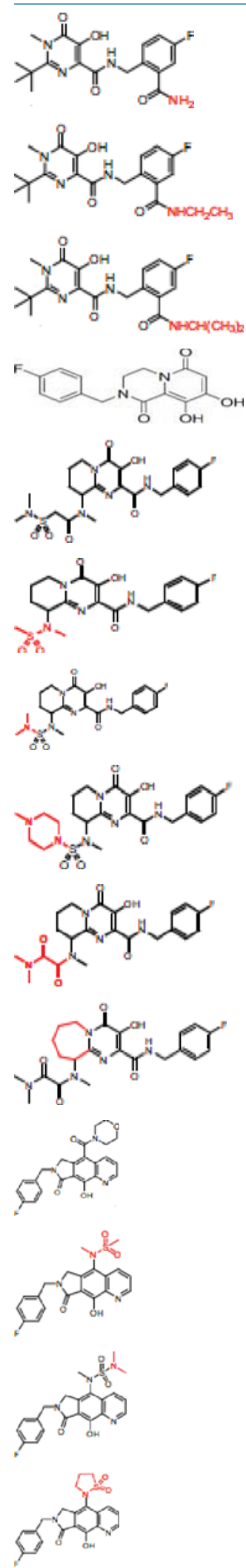

1.11

descriptors for the 26 Elvitegravir drug analogs [Table 1]. After calculation of the descriptors for all molecules and multiple linear regression analysis by the MINITAB 14 tool the following best three mathematical equations were derived.

$\log$ IC $50=4.71+0.0116 \mathrm{MW}-0.0514 \mathrm{MV}-0.0911$ LogP - 0.0741 WI - 9.67 SMTI (Eq.1)

$\log$ IC $50=1.47+0.0130 \mathrm{MW}-0.0481 \mathrm{MV}-0.101$ LogP - 0.0701 WI + 0.106 TIE (Eq.2)

$\log$ IC $50=5.18+0.0108$ MW -0.0564 MV -0.0660 WI - 11.4 SMTI + 0.022 TIE (Eq.3)

The statistics from all three models exhibit the dependency to both structural and physiochemical descriptors are presented in the above equations. Inorder to confirm our results Log IC50 values were predicted from the above equations and results were compared to previously calculated ones. Such correlations for the above three equations have been given in Figures 1-3 respectively.

\section{DISCUSSION}

In general the topological and structural descriptors are very important types of molecular descriptors for bioactivity prediction. ${ }^{[13]}$ However, in our multiple linear regression analysis it was observed that among the above descriptors the structural descriptors are less important for anti-HIV activities. All the statistics of the equations have been considered to exhibit the structural and physiological parameters to model the Log IC50 value of the drug analogs. Comparing the variance value (R-Sq) among the equations [Table 2], the variances decrease when more independent variables (descriptors) for the structural type were considered. For the same set of descriptors (Eq.1 and Eq.2) only two combinations of structural descriptors showed less change in variance value (R-Sq), however, when three structural descriptors (Eq.3) were considered, the variance value were observed as it decreases significantly. Also, $P$ value for regression was observed to be 0.000 in all the selected models in Minitab calculations. Since the value is less than the level of signicance (0.005) so it indicates the validity of the considered descriptors. Further, 3D descriptor calculation and QSAR model building would provide the features that significantly contribute to the physiochemical property which is related to the activity of the drug. Our results show that the anti-HIV activity of the Elvitegravir derivatives can be successfully modeled with some selected physiochemical and structural descriptors. In this method, the reliable prediction obtained can be successfully used for determining the anti-HIV activity of drugs. 
Table 2: Calculation of statistical parameters for the best model consideration

\begin{tabular}{lccc}
\hline Model number & R-Sq value (\%) & F value & $P$ value \\
\hline 1 & 76.7 & 13.86 & 0.000 \\
2 & 73.1 & 11.40 & 0.000 \\
3 & 69.7 & 9.66 & 0.000 \\
\hline
\end{tabular}

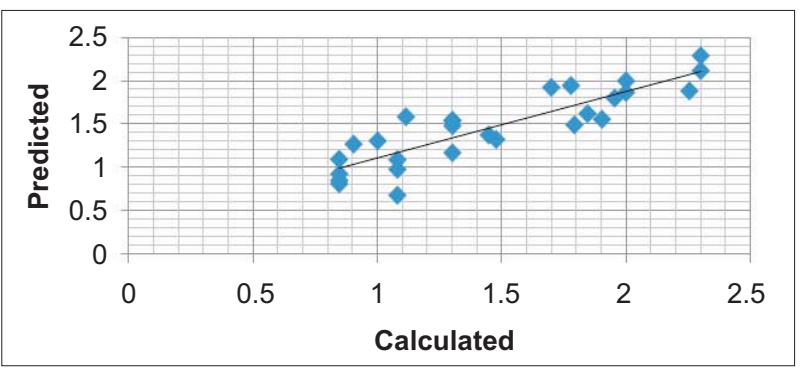

Figure 1: Plot between calculated and predicted value for Log IC50 (Model 1)

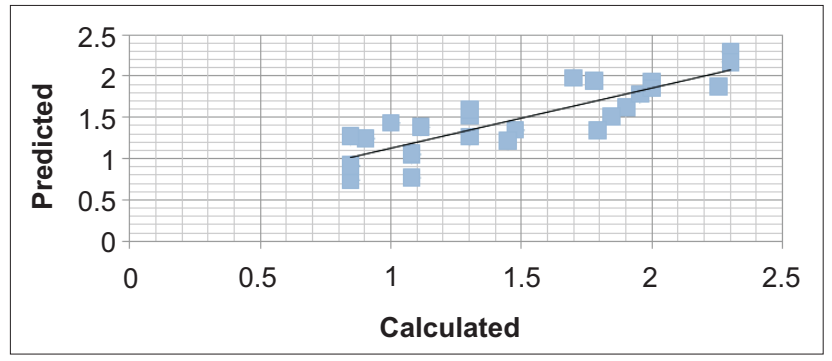

Figure 2: Plot between calculated and predicted value for Log IC50 (Model 2)

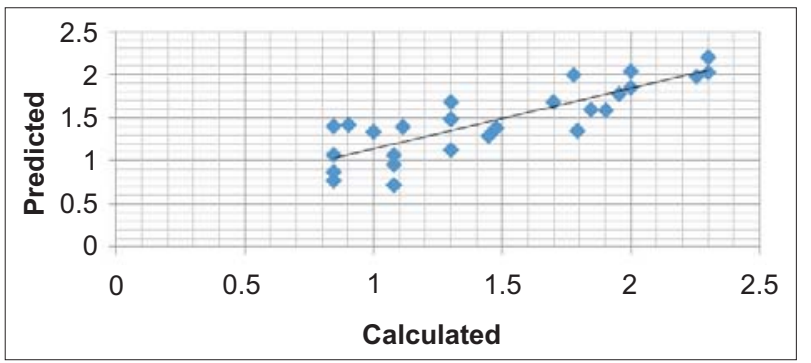

Figure 3: Plot between calculated and predicted value for Log IC50 (Model 3)

\section{ACKNOWLEDGMENT}

We are thankful to Chief Executive, Majhighariani Institute of Technology and Science, Ryagada for providing us the MIRC lab for computing facility.

\section{REFERENCES}

1. Ivai.org.in (Homepage on the internet). India: International Aids Vaccine initiative. Available from: http://www.iavi.org.in/sc_research.html. [Last accessed on 2010 Jun 15].

2. De Clercq E. Anti-HIV drugs: 25 compounds approved within 25 years after the discovery of HIV. Int J Antimicrob Agents 2008;33:307-20.

3. Delelis O, Carayon K, Saib A, Deprez E, Mouscadet JF. Integrase and integration: Biochemical activities of HIV-1 integrase. Retrovirology 2008;5:114.

4. Asante-Appiah E, Skalka AM. HIV-1 integrase: Structural organization, conformational changes, and catalysis. In: Rlaramorosch K, Murphy FA, Shawn AJ, editors. Adv Virus Res. Academic Press; 1999. p. 351-69.

5. Klibanov OM. Elvitegravir, an oral HIV integrase inhibitor, for the potential treatment of HIV infection. Curr Opin Investig Drugs 2009;10:190-200.

6. Zolopa AR, Berger DS, Lampiris H, Zhong L, Chuck SL, Enejosa JV, et al. Activity of elvitegravir, a once-daily integrase inhibitor, against resistant HIV Type 1: Results of a phase 2, randomized, controlled dose-ranging clinical trial. J Infect Dis 2010;201:814-22.

7. Winkler DA. The role of quantitative structure - activity relationships (QSAR) in biomolecular discovery. Brief Bioinform 2002;3:73-86.

8. Serrao E, Odde S, Ramkumar K, Neamati N. Raltegravir, elvitegravir, and metoogravir: The birth of "me-too" HIV-1 integrase inhibitors. Retrovirology 2009;6:25.

9. Chemaxon.com (Home page on the internet). Marvin calculator plugin and chemical terms demo.Inc; c1999-2010. Available from: http://www. chemaxon.com/marvin/sketch/index.jsp. [Last accessed on 2010 Jun 15].

10. Schuettelkopf AW, van Aalten DM. PRODRG - a tool for highthroughput crystallography of protein-ligand complexes. Acta Crystallogr 2004;D60:1355-63.

11. Tetko IV. Computing chemistry on the web. Drug Discov Today 2005;10:1497-500.

12. Wild DJ. MINITAB Release 14. J Chem Inf Model 2005;45:212.

13. Du Q, Mezey PG, Chou KC. Heuristic molecular lipophilicity potential (HMLP): A 2D-QSAR study to LADH of molecular family pyrazole and derivatives. J Comput Chem 2005;26:461-70.

How to cite this article: Satpathy R, Ghosh S. In-silico comparative study and quantitative structure-activity relationship analysis of some structural and physiochemical descriptors of elvitegravir analogs. J Young Pharmacists $2011 ; 3: 246-9$

Source of Support: Nil, Conflict of Interest: None declared. 